\title{
Mechanically ventilated patients exhibit decreased particle flow in exhaled breath as compared to normal breathing patients
}

\author{
Ellen Broberg ${ }^{1}$, Jesper Andreasson ${ }^{2}$, Mohammed Fakhro $^{2}$, Anna-Carin Olin ${ }^{3}$, \\ Darcy Wagner $\mathbb{1}^{4,5,6}$, Snejana Hyllén ${ }^{1}$ and Sandra Lindstedt (102,5
}

Affiliations: ${ }^{1}$ Dept of Cardiothoracic Anaesthesia and Intensive Care, Skåne University Hospital, Lund University, Lund, Sweden. ${ }^{2}$ Dept of Cardiothoracic Surgery, Skåne University Hospital, Lund University, Lund, Sweden. ${ }^{3}$ Occupational and Environmental Medicine, Dept of Public Health and Community Medicine, Institute of Medicine, Sahlgrenska Academy, University of Gothenburg, Gothenburg, Sweden. ${ }^{4}$ Lund University, Experimental Medical Sciences, Lung Bioengineering and Regeneration, Lund, Sweden. ${ }^{5}$ Wallenberg Centre for Molecular Medicine, Lund University, Lund, Sweden. ' Lund Stem Cell Center, Lund University, Lund, Sweden.

Correspondence: Sandra Lindstedt, Dept of Cardiothoracic Surgery and Transplantation, Skåne University Hospital, SE-221 85 Lund, Sweden. E-mail: sandra.lindstedt_ingemanssonamed.lu.se

\section{ABSTRACT}

Introduction: In this cohort study, we evaluated whether the particles in exhaled air (PExA) device can be used in conjunction with mechanical ventilation during surgery. The PExA device consists of an optical particle counter and an impactor that collects particles in exhaled air. Our aim was to establish the feasibility of the PExA device in combination with mechanical ventilation (MV) during surgery and if collected particles could be analysed. Patients with and without nonsmall cell lung cancer (NSCLC) undergoing lung surgery were compared to normal breathing (NB) patients with NSCLC.

Methods: A total of 32 patients were included, 17 patients with NSCLC (MV-NSCLC), nine patients without NSCLC (MV-C) and six patients with NSCLC and not intubated (NB). The PEx samples were analysed for the most common phospholipids in surfactant using liquid-chromatography-massspectrometry (LCMS).

Results: MV-NSCLC and MV-C had significantly lower numbers of particles exhaled per minute (particle flow rate; PFR) compared to NB. MV-NSCLC and MV-C also had a siginificantly lower amount of phospholipids in PEx when compared to NB. MV-NSCLC had a significantly lower amount of surfactant A compared to NB.

Conclusion: We have established the feasibility of the PExA device. Particles could be collected and analysed. We observed lower PFR from MV compared to NB. High PFR during MV may be due to more frequent opening and closing of the airways, known to be harmful to the lung. Online use of the PExA device might be used to monitor and personalise settings for mechanical ventilation to lower the risk of lung damage.

@ERSpublications

The PExA device is safe to use in conjunction with mechanical ventilation during surgery, and can measure and collect particles in exhaled air for subsequent biochemical analysis http://bit.ly/ 2ofo6gw

Cite this article as: Broberg E, Andreasson J, Fakhro M, et al. Mechanically ventilated patients exhibit decreased particle flow in exhaled breath as compared to normal breathing patients. ERJ Open Res 2020; 6: 00198-2019 [https://doi.org/10.1183/23120541.00198-2019].

Please contact the author for data requests.

Received: 11 Aug 2019 | Accepted after revision: 17 Oct 2019

Copyright @ERS 2020 This article is open access and distributed under the terms of the Creative Commons Attribution Non-Commercial Licence 4.0. 


\section{Introduction}

Patients undergoing lung surgery are mainly those with nonsmall cell lung cancer (NSCLC). Patients with other lung pathologies such as pneumothorax and infections are also considered for lung surgery. During lung surgery, patients are often ventilated using one-lung ventilation (OLV) since the affected lung is not ventilated in order to facilitate surgery.

In the present study we aimed to establish the feasibility of the particles in exhaled air (PExA) device in conjunction with mechanical ventilation during surgery. The PExA device has been studied previously in normal breathing (NB) patients but has not yet been used in combination with mechanical ventilation during surgery [1-3]. The PExA device consists of an optical particle counter that can measure number and size of exhaled particles (PEx) with an impactor where the particles are sampled on a membrane for subsequent biochemical analysis. PEx are thought to originate from the respiratory tract lining fluid (RTLF) that covers the epithelial wall of the airways [4-8]. In awake patients, here described as normal breathing, a special breathing manoeuvre is used, to reinforce airway closure and re-opening [9]. Previously it has been shown that a change in the composition of the RTLF reflects different airway diseases $[1,2,10-13]$.

Conventional monitoring during surgery is unable to detect subtle changes or stressors in the lung which precede the onset of lung injury. New and ideally noninvasive techniques which can detect early changes in the lung and can be used to optimise mechanical ventilation would be a step forward towards creating personalised ventilation treatments and hopefully reducing ventilator induced lung injury. Our aims for this study were to: evaluate the feasibility and safety of using the PExA device during surgery on intubated mechanically ventilated patients, and to ascertain if differences in the composition of exhaled particles can be detected between mechanically ventilated patients and NB patients.

\section{Methods}

The study was performed in accordance with the Declaration of Helsinki and was approved by the local ethics committee (Dnr 2017/519). All patients signed a written, informed consent before entering the study. The study conducted according to the STROBE statement. There was no bias applicable in the study.

\section{Patient demographics}

A total of 32 patients were included and all patients signed a written informed consent. 26 patients were included in the mechanical ventilation cohort and were subsequently divided into two cohorts; 17 patients with NSCLC (MV-NSCLC) and nine patients without NSCLC (MV-C). Another group of six patients with NSCLC who were not intubated was also included (NB). A flow chart is shown in figure 1 and demographic data are shown in table 1. For MV-NSCLC and MV-C, measurements were started after the patient was in correct position for surgery and on OLV. The measurements stopped at the end of surgery when the patients were still on OLV. For a total of nine patients, surgery allowed measurements directly following OLV for a period of 5 min during double-lung ventilation (DLV). A time chart is seen in figure 2.

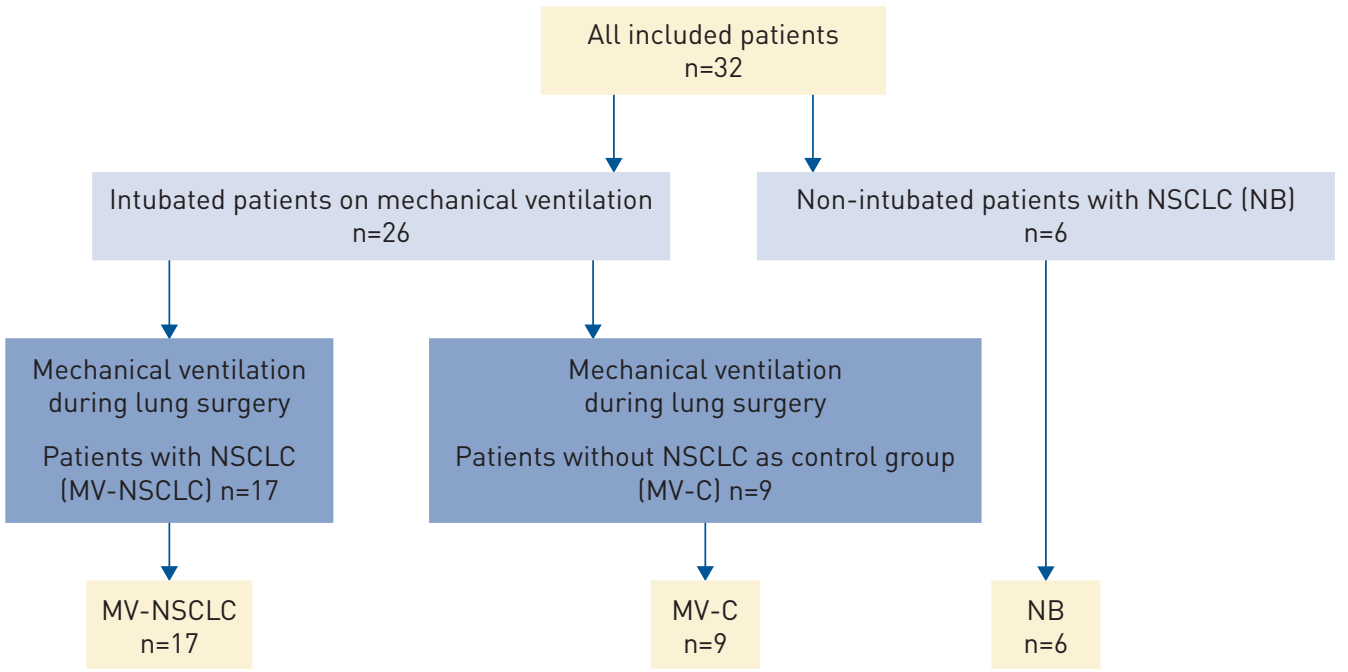

FIGURE 1 Mechanical ventilation during lung surgery divided into mechanical ventilation in nonsmall cell-lung cancer patients (MV-NSCLC) and mechanical ventilation in control patients (MV-C). The third group is nonintubated, normal breathing (NB) patients. 


\section{TABLE 1 Demographics for the three different groups}

\begin{tabular}{|c|c|c|c|}
\hline & MV-NSCLC & MV-C & NB \\
\hline Age years & $68 \pm 2$ & $60 \pm 5$ & $71 \pm 3$ \\
\hline Females & $9(50)$ & $5(56)$ & 2 (33) \\
\hline $\mathrm{BMI} \mathrm{kg} \cdot \mathrm{m}^{-2}$ & $27 \pm 1.5$ & $27 \pm 2.4$ & $27 \pm 2.4$ \\
\hline Smoking & 14 (78) & $3(33)$ & $4(67)$ \\
\hline COPD & $2(11)$ & 0 & 2 (33) \\
\hline$V_{\mathrm{T}} \mathrm{L}$ & $3.42 \pm 0.18$ & $3.86 \pm 0.25$ & $3.20 \pm 0.37$ \\
\hline$V_{\mathrm{T}} \%$ & $85 \pm 5$ & $84 \pm 3$ & $79 \pm 8$ \\
\hline $\mathrm{FEV}_{1} \mathrm{~L}$ & $2.45 \pm 0.16$ & $2.15 \pm 0.18$ & $2.18 \pm 0.27$ \\
\hline FEV $_{1} \%$ & $86 \pm 5$ & $74 \pm 4$ & $74 \pm 8$ \\
\hline$D_{\text {Lco }} \%$ & $84 \pm 4$ & $80 \pm 6$ & $72 \pm 5$ \\
\hline \multicolumn{4}{|c|}{$\begin{array}{l}\text { Data are presented as mean } \pm \text { SEM or } n(\%) \text {. MV-NSCLC: mechanical ventilation in nonsmall cell lung cancer } \\
\text { patients; } M V-C \text { : mechanical ventilation in control patients; NB: normal breathing patients; } \mathrm{BMI} \text { : body mass } \\
\text { index; } V_{\mathrm{T}} \text { : tidal volume; } \mathrm{FEV}_{1} \text { : forced expiratory volume in } 1 \mathrm{~s} ; D_{\mathrm{LCO}} \text { : diffusing capacity of the lung for carbon } \\
\text { monoxide. }\end{array}$} \\
\hline
\end{tabular}

\section{Anaesthesia and ventilation for patients undergoing lung surgery}

Anaesthesia during surgery was performed according to standard procedure with target controlled infusion (TCI). TCI was performed with remifentanil $50 \mu \mathrm{g} \cdot \mathrm{mL}^{-1}$ (ampules $2 \mathrm{mg} \cdot \mathrm{mL}^{-1}$; Actavis Group PTC ehf., Hafnarfjordur, Iceland) using the Minto model and Propofol-Lipuro $20 \mathrm{mg} \cdot \mathrm{mL}^{-1}$ (B. Braun; Melsungen AG, Melsungen, Germany) using the Marsh model. The muscle relaxant used was Esmeron (Rocuronium) $10 \mathrm{mg} \cdot \mathrm{mL}^{-1}$ (MSD, Haarlem, Netherlands), and Bridion (Sugammadex) $100 \mathrm{mg} \cdot \mathrm{mL}^{-1}$ (MSD, Hoddesdon, UK) was used to reverse the muscle relaxation effect at the end of surgery. Oral intubation was performed using a double-lumen endotracheal tube, Rüsch Bronchopart Teleflex medical (Westmeath, Ireland) or VivaSight DL AS (Ambu, Ballerup, Denmark). Mechanical ventilation was performed using volumecontrolled pressure-support with a Maquet ventilator FLOW-i (Getinge Group, Solna, Sweden). The ventilator settings were according to local guidelines: tidal volume of $6-8 \mathrm{~mL} \cdot \mathrm{kg}^{-1}$, minimum positive end-expiratory pressure (PEEP) of $5 \mathrm{cmH}_{2} \mathrm{O}$, end-inspiratory pressures $<25 \mathrm{cmH}_{2} \mathrm{O}$, and target $\mathrm{CO}_{2}$ levels of 4.6 to $6 \mathrm{kPa}$.

\section{PEXA measurements during mechanical ventilation}

The PExA 2.0 device (PExA, Gothenburg, Sweden) contain an optical particle counter (OPC) connected to an impactor for collection of $\operatorname{PEx}[9,10,14]$. PEx in the diameter range of $0.41-4.55 \mu \mathrm{m}$ can be measured continuously using the OPC and the impactor samples particles in the size range $0.4-7.2 \mu \mathrm{m}$. In the present study, the PExA 2.0 instrument was customised to be able to be used in conjunction with mechanical ventilation as we have described previously in preclinical in vivo settings $[14,15]$. The device was connected to the outflow air of the mechanical respiratory circuit, as seen in figure 3. During surgery, the PExA device measured number of particles (count) and total accumulated mass (ng) of particles from

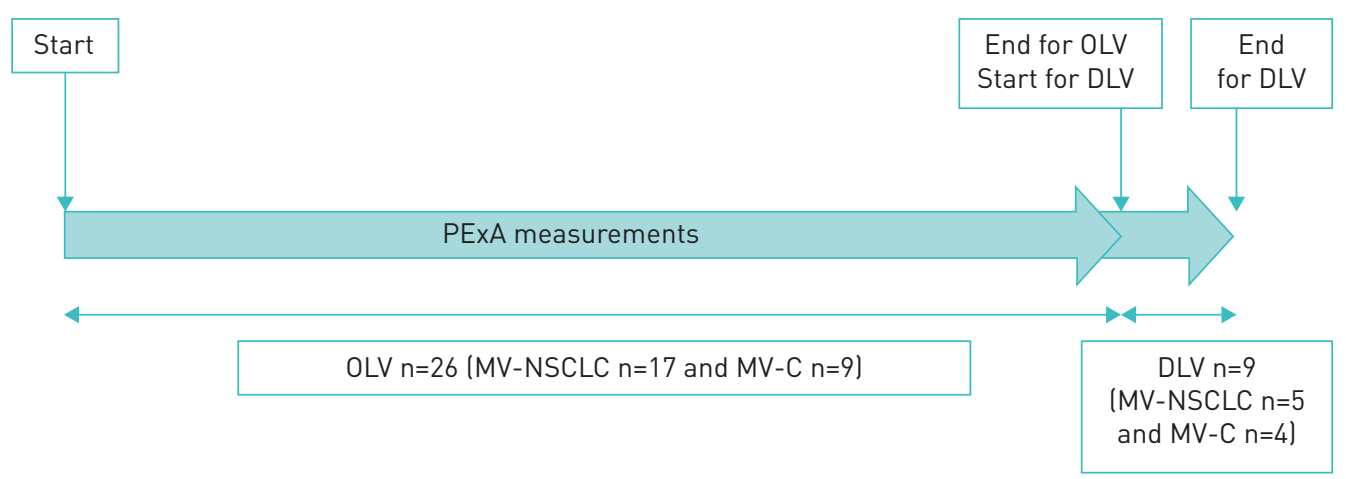

FIGURE 2 A timeline of the experiment. Measurements were started when the patients were in the correct position and on one-lung ventilation (OLV). The measurements stopped at the end of surgery when the patients were still on OLV. For nine patients, surgery allowed a period of 5 mins for measurements to be made when changing directly from OLV to double-lung ventilation (DLV) before ending the surgery. PeXA: particles in exhaled air. 
FIGURE 3 The respiratory circuit. The yellow arrow shows the balloon representing the patient and the black arrow, the non-rebreathing valve. The red arrows shows the direction of airflow from the mechanical ventilator to the balloon representing the patient and further on to the particles in exhaled air (PExA) device. The blue arrows show the direction of airflow from the PExA device back to the ventilator.

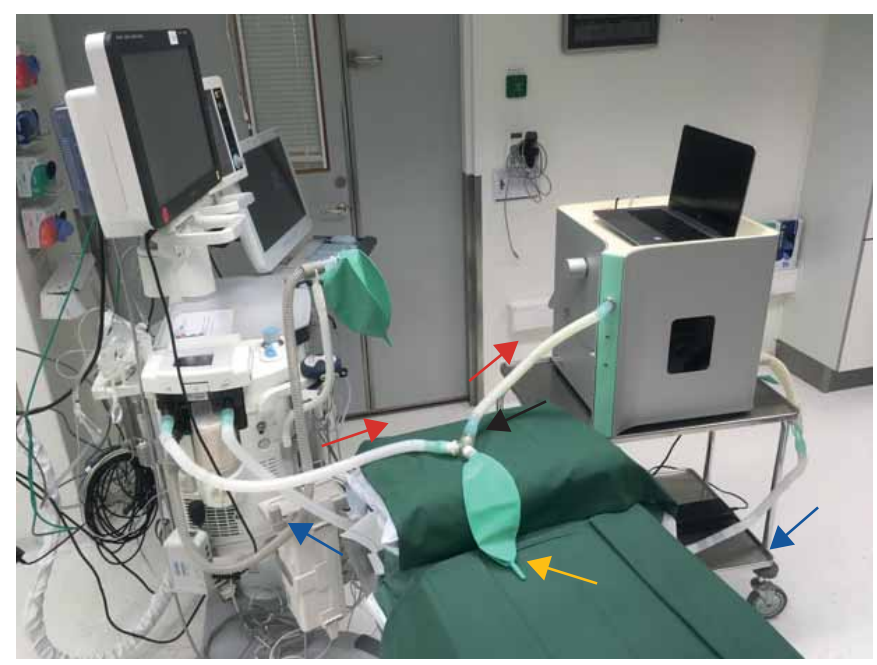

the airways. Number of particles are described as particles per minute, named particle flow rate (PFR). Particles were collected onto a membrane for biochemical analysis and referred to as PEx. During lung surgery, the affected lung was disconnected from the mechanical ventilation to optimise the removal of affected lung parenchyma. Measurements were first performed during OLV. Then, for nine patients in total from both MV-NCSLC and MV-C, an additional collection period of 5 mins occurred at the end of surgery as the patients transitioned from OLV to DLV.

Particle collection in non-intubated, awake, NB patients

PEx were collected in the NB group using a previously published and validated PExA method with a breathing manoeuvre for patients breathing spontaneously $[9,12,13,16,17]$.

\section{Particle collection and membrane preparation with the PEXA device}

Particles in exhaled air were collected using a two-stage inertial impactor, described in previous articles $[9,10,14]$. Mass spectrometry was used to quantify the phospholipids di-palmitoyl-phosphatidyl-choline (DPPC) and palmitoyl-oleoyl-phosphocholine (POPC). The method is described in previous articles $[2,15]$. Albumin and SP-A were analysed by ELISA, described in previous articles $[12,13,16,17]$.

Out of the 26 mechanically ventilated patients, only 15 patients reached a collected mass of $\geqslant 50 \mathrm{ng}$. Only membranes with a collected mass of $\geqslant 50 \mathrm{ng}$ were analysed. Due to the low collected mass, the rest of the membranes were not analysed. In all NB patients the PEx collection stopped when 100 ng was reached whereas the surgery time was a limiting factor among the mechanical ventilated patients. Membranes containing a total collected mass above $100 \mathrm{ng}$ were divided into two and were sent for both phospholipid and protein analysis. The remaining membranes were randomised into either phospholipid or protein analysis. Among the mechanically ventilated patients 13 samples were analysed for DPPC and POPC. All of the samples reached the detection level. 11 samples from the mechanical ventilated patients were analysed for albumin where nine of the samples reached the detection level. Furthermore, 11 samples were analysed for SP-A where eight samples reached the detection levels. Among NB patients six samples were analysed for DPPC and POPC and six samples were analysed for albumin and SP-A. All samples from the NB patients reached detection levels.

\section{Calculations and statistics}

A power calculation was done based on biochemical analysis results in prior studies on normal breathing patients $[1,2,9,13]$. Descriptive statistics, in the form of the number of patients, mean, and the standard error of the mean for the different haemodynamic parameters were analysed. Results of the analysed particles in exhaled air are shown as median with confidence interval (5-95\%). Statistically significant differences between the different groups were tested with Mann-Whitney and differences within the groups were tested with the Wilcoxon signed-rank test. All statistical analysis was performed using Graf Pad Prism Version 8 (USA). Significance was defined as $\mathrm{p}<0.001\left(^{* * *}\right), \mathrm{p}<0.01\left(^{* *}\right), \mathrm{p}<0.05\left(^{*}\right)$ and $\mathrm{p}>0.05$ and not significant (NS). 


\section{Results}

PFR and particles per mass

PFR were 942 (388-4898) in the MV-NSCLC group, 1655 (968-5753) in the MV-C group and 10520 (6933-13 462) in the NB group. Comparing the groups, a significant difference was found in PFR between MV-NSCLC and NB $(p=0.036)$ and between MV-C and NB $(p=0.0004)$ but no significance was seen between MV-NSCLC and MV-C, shown in figure 4a. Average particle mass were $0.148 \times 10^{-3} \mathrm{ng}$ $\left(0.128 \times 10^{-3}-0.196 \times 10^{-3}\right)$ in the MV-NSCLC group, $0.155 \times 10^{-3} \mathrm{ng}\left(0.095 \times 10^{-3}-0.222 \times 10^{-3}\right)$ in the MV-C group and $0.433 \times 10^{-3} \mathrm{ng}\left(0.387 \times 10^{-3}-0.700 \times 10^{-3}\right)$ in the NB group. Comparing the groups, a significant difference was found in average particle mass between MV-NSCLC and NB $(p=0.0004)$ and between MV-C and NB ( $\mathrm{p}=0.0004)$ but no significance was seen between MV-NSCLC and MV-C $(p=0.92)$, shown in figure $4 b$.

\section{Biochemical collection of phospholipids and proteins}

DPPC was 2.50 (1.80-5.10) wt. \% in the MV-NSCLC group, 2.10 (1.30-2.70) wt. \% in the MV-C group and 8.35 (4.80-9.90) wt. \% in the NB group, with significant differences between MV-NSCLC and NB ( $p=0.001)$, MV-C and NB ( $p=0.004)$ but not for MV-NSCLC and MV-C $(p=0.102)$, as shown in figure 5a. POPC was $0.65(0.40-1.30)$ wt. \% in the MV-NSCLC group, $0.60(0.30-0.70)$ wt. \% in the MV-C group and 2.05 (1.10-2.40) wt. \% in the NB group, with significant difference between MV-NSCLC and NB (0.001), MV-C and NB (0.004) but not for MV-NSCLC and MV-C (0.493), as shown in figure 5b. DPPC and POPC ratio was 3.965 (3.03-4.70) in the MV-NSCLC group, 3.76 (2.70-4.04) in the MV-C group and 4.22 (3.88-4.59) in the NB group. Significance could be seen between MV-C and NB ( $p=0.030)$ but not between MV-NSCLC and MV-C ( $\mathrm{p}=0.222)$ or between MV-NSCLC and NB ( $\mathrm{p}=0.491)$, as seen in figure $5 \mathrm{c}$.

For albumin and SP-A unfortunately two samples from the MV-C group did not reach detection levels and therefore we only present MV-NSCLC and NB for albumin and Sp-A. Albumin was $2.01(0.77-4.44)$ wt. \% in the MV-NSCLC group and $2.27(1.59-7.05)$ wt. \% in the NB group ( $p=0.485)$ and SP-A in the MV-NSCLC group was 0.57 (0.11-0.94) weight per cent protein (wt. \%) and $2.16(0.30-3.41)$ wt. \% in the NB group $(\mathrm{p}=0.024)$ as shown in figure $5 \mathrm{~d}$ and e. Albumin and SP-A ratio was $4.35(1.89-8.11)$ in the MV-NSCLC group and $1.51(0.47-6.81)$ in the NB group $(\mathrm{p}=0.041)$, as shown in figure $5 f$.

\section{One-lung ventilation and double-lung ventilation during lung surgery}

During mechanical ventilation in lung surgery, measurements were possible in a total of nine out of 26 patients when going from OLV to DLV. During OLV, PFR was 119 (29-1930) compared to DLV 171 (30-2239) ( $\mathrm{p}=0.002)$ as shown in figure 6a. PFR was 157 (18-3403) during OLV and 171 (24-4348) during DLV in the MV-NSCLC group ( $\mathrm{p}=0.7)$, and 83 (29-274) during OLV and 316 (30-1955) during DLV in
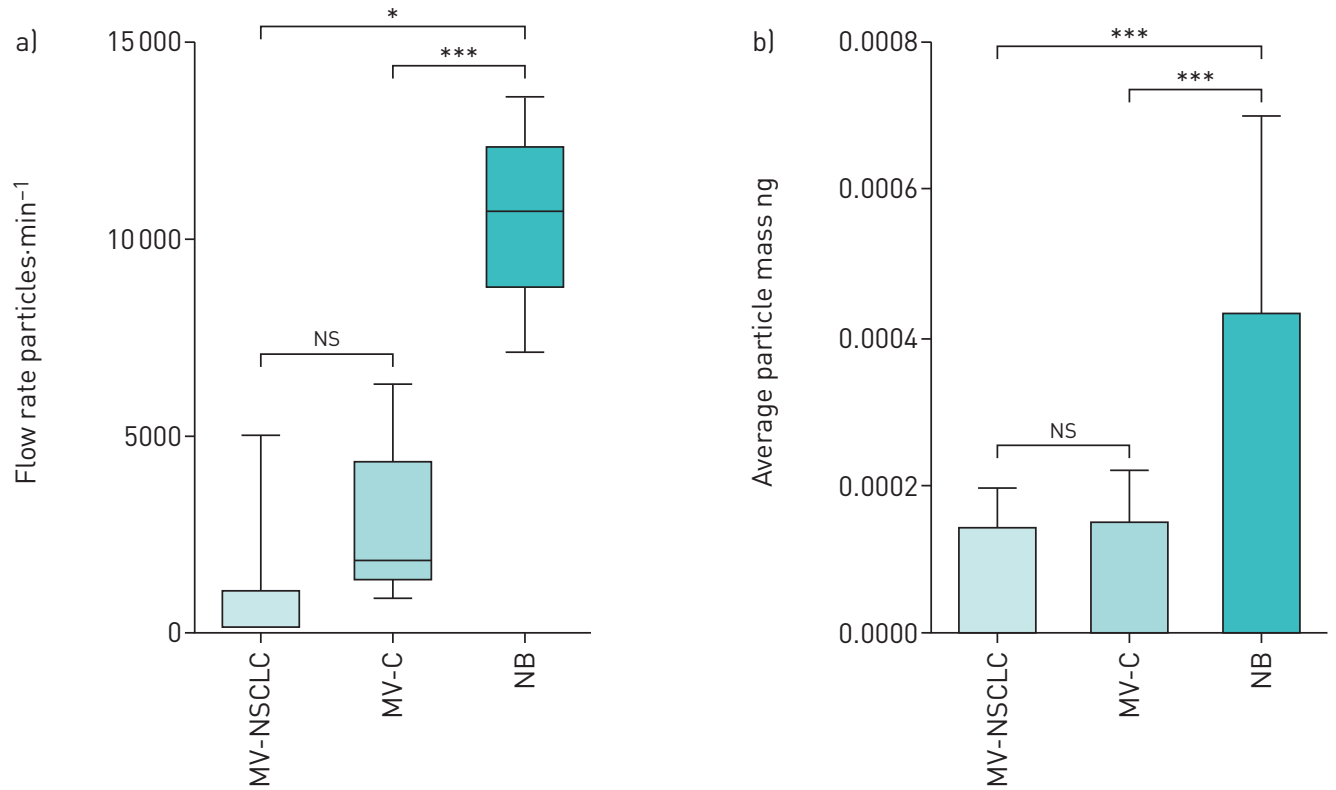

FIGURE 4 a) Particle flow rate exhaled per minute in mechanical ventilation in nonsmall cell lung cancer patients (MV-NSCLC), mechanical ventilation in control patients (MV-C) and normal breathing patients (NB). b) Exhaled average particle mass in ng in MV-NSCLC, MV-C and NB. NS: nonsignificant. *: $p<0.05 ;{ }^{* * *}$ : $p<0.001$. 

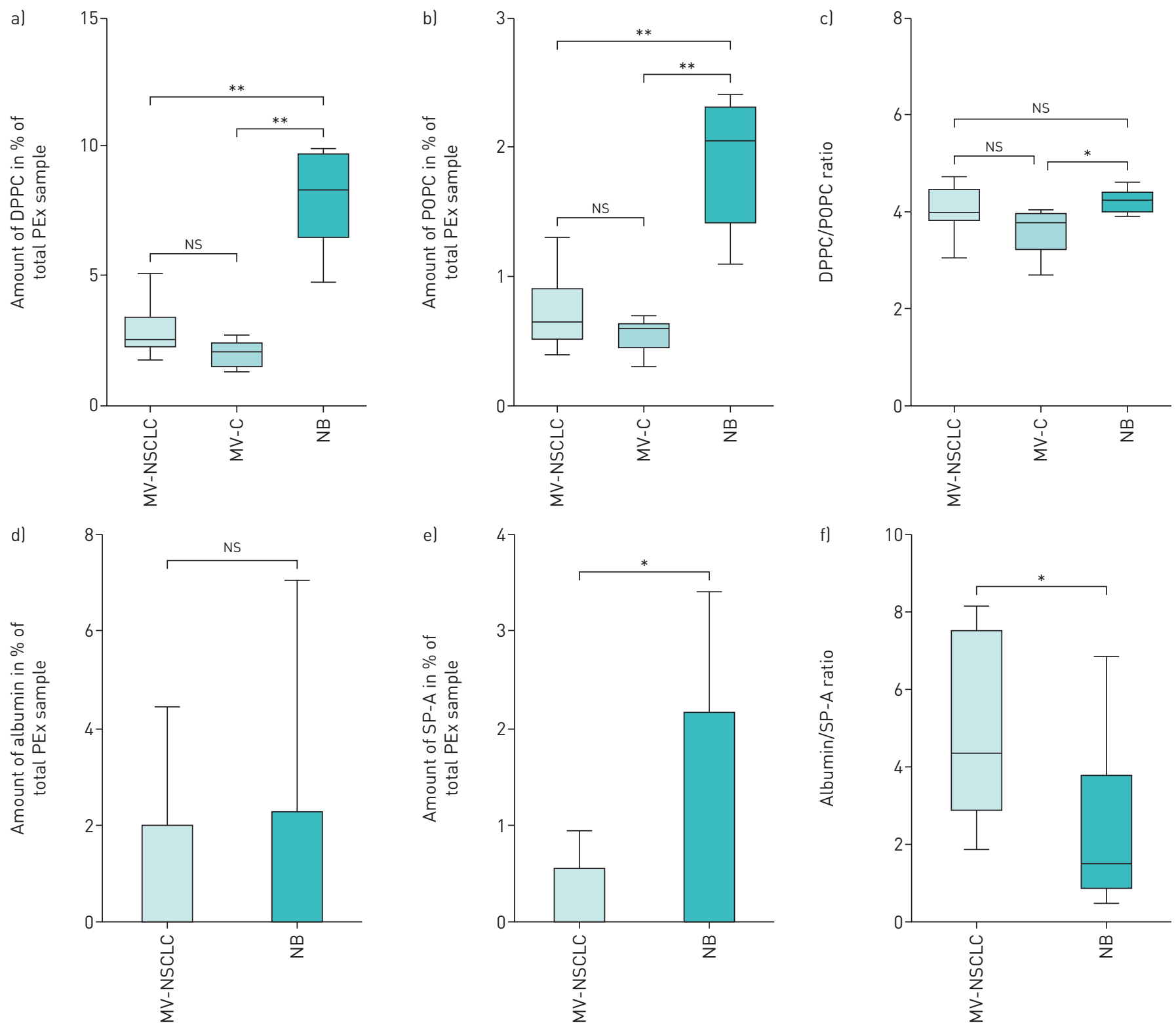

FIGURE 5 a) Weight per cent protein (wt. \%) of total PEx sample for phospholipids di-palmitoyl-phosphatidyl-choline (DPPC) comparing nonsmall cell lung cancer patients on mechanical ventilation (MV-NSCLC), control patients on mechanical ventilation (MV-C) and non-intubated normal breathing patients (NB). b) wt. \% of total PEx sample for palmitoyl-oleoyl-phosphocholine (POPC) comparing MV-NSCLC, MV-C and NB. c) Ratio of DPPC/POPC between MV-NSCLC, MV-C and NB. d) wt. \% of total PEx sample for surfactant A (SP-A) comparing MV-NSCLC and NB patients. e) wt. \% of total PEx sample for albumin comparing MV-NSCLC and NB. f) The albumin/SP-A ratio between MV-NSCLC and NB. NS: nonsignificant. ${ }^{*}: \mathrm{p}<0.05 ;{ }^{* *}: \mathrm{p}<0.01$.

the MV-C group $(\mathrm{p}=0.3)$. During OLV, average particle mass was $0.399 \times 10^{-3}\left(0.268 \times 10^{-3}-0.834 \times 10^{-3}\right)$ compared with DLV $0.604 \times 10^{-3}\left(0.368 \times 10^{-3}-0.678 \times 10^{-3}\right)(\mathrm{p}=0.5)$ as shown in figure $6 \mathrm{~b}$.

\section{Haemodynamics}

Haemodynamics and mechanical ventilation parameters in the different groups are shown in table 2. Peak pressure was statistically different during MV-NSCLC but not for any other parameters, as seen in table 2.

\section{Discussion}

We have shown that the PExA device can safely be used during surgery. The study also demonstrates that particle profiles for both PFR and mass from the airways can be measured in patients undergoing lung surgery and that there are noticeable differences between mechanically ventilated and NB patients. The particle composition of the samples was analysed regarding phospholipids and proteins; the latter was 

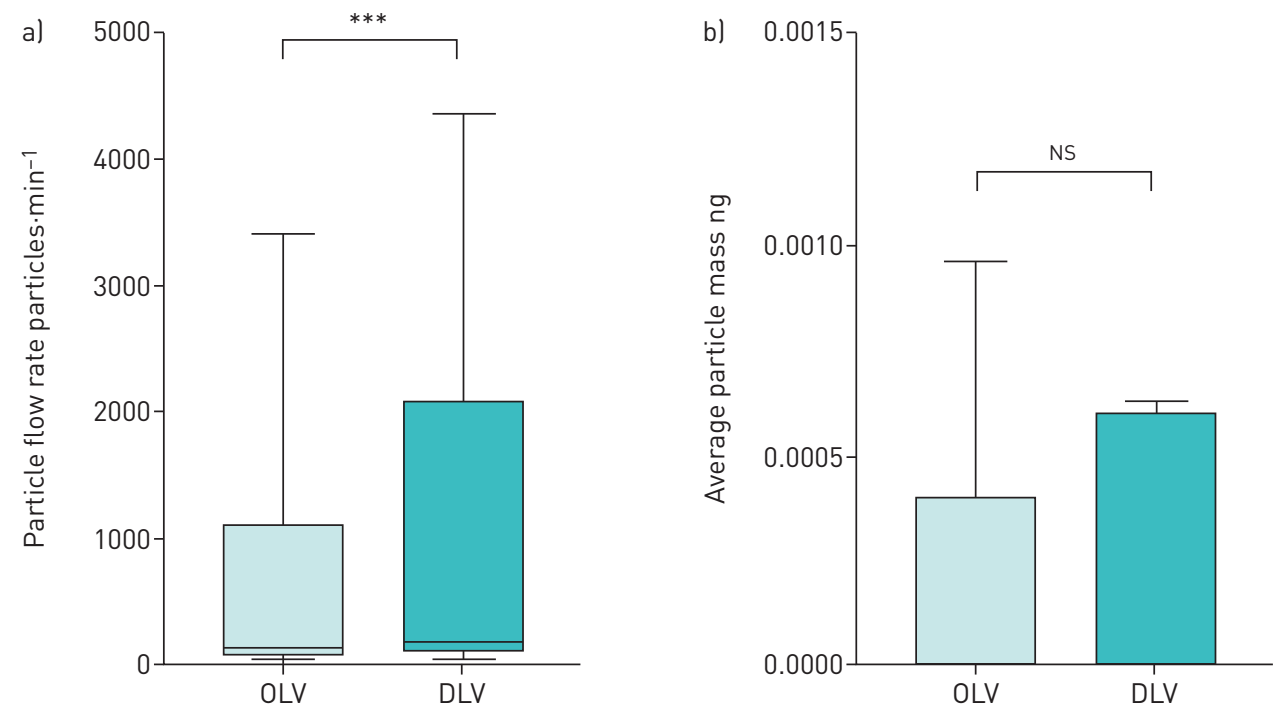

FIGURE 6 a) Particle flow rate exhaled per minute measured for 5 mins of one-lung ventilation (OLV) before opening up the other lung and followed by measurements for $5 \mathrm{~min}$ of double-lung ventilation (DLV). b) Exhaled average particle mass in ng measured for $5 \mathrm{~min}$ of OLV followed by measurements for 5 min of DLV. NS: nonsignificant. ${ }^{* * *}$ : $p<0.001$.

a challenge in the samples from mechanical ventilated patients due to the low masses. However, $100 \%$ of the analysed samples for phospholipids reached detection levels whereas only $73 \%$ of the analysed samples for proteins reached detection levels. Furthermore, all the samples from NB reached detection levels. When comparing mechanical ventilated patients, patients with lung cancer (MV-NSCLC) from those under surgery $(\mathrm{MV}-\mathrm{C})$ there was a trend towards lower amount of exhaled particles, it did however not reach statistical significance.

\section{NB patients generate higher PFR and higher average mass compared to mechanically} ventilated patients

We found that NB patients generated a considerably higher PFR and higher average particle mass compared to patients on mechanical ventilation. One of the potential reasons for this difference may be due to differences in the opening and closing of the airways between the two different breathing modes. The breathing manoeuvre in NB group uses large tidal volumes and the aim is to provoke an opening and closing of the distal airways. On the other hand, during mechanical ventilation for lung surgery, patients are on muscle relaxants and PEEP is applied with the primary aim of keeping the distal airways open to

TABLE 2 Haemodynamics during mechanical ventilation in nonsmall cell lung cancer (MV-NSCLC) and mechanical ventilation in the control group (MV-C)

\begin{tabular}{|c|c|c|c|c|c|c|}
\hline \multirow[t]{2}{*}{ Parameters } & \multicolumn{3}{|c|}{ MV-NSCLC } & \multicolumn{3}{|c|}{ MV-C } \\
\hline & Start & Stop & p-value & Start & Stop & p-value \\
\hline BP systolic $\mathrm{mmHg}$ & $106 \pm 3$ & $104 \pm 2$ & 0.65 & $102 \pm 5$ & $104 \pm 2$ & 0.87 \\
\hline BP diastolic $\mathrm{mmHg}$ & $59 \pm 2$ & $57 \pm 1$ & 0.24 & $59 \pm 3$ & $59 \pm 2$ & 0.98 \\
\hline MAP mmHg & $76 \pm 2$ & $73 \pm 1$ & 0.23 & $75 \pm 3$ & $76 \pm 3$ & 0.78 \\
\hline Pulse & $76 \pm 3$ & $71 \pm 4$ & 0.41 & $78 \pm 4$ & $74 \pm 4$ & 0.41 \\
\hline $\mathrm{EtCO}_{2} \mathrm{kPa}$ & $5.1 \pm 0.1$ & $4.7 \pm 0.1$ & 0.11 & $5.3 \pm 0.2$ & $4.8 \pm 0.2$ & 0.14 \\
\hline Saturation \% & $99 \pm 1$ & $99 \pm 1$ & 0.15 & $99 \pm 1$ & $99 \pm 1$ & 0.1 \\
\hline Respiration frequency & $15 \pm 1$ & $16 \pm 1$ & 0.61 & $16 \pm 1$ & $17 \pm 1$ & 0.84 \\
\hline$V_{\mathrm{T}}$ inspiration $\mathrm{mL}$ & $453 \pm 15$ & $470 \pm 13$ & 0.43 & $437 \pm 27$ & $418 \pm 27$ & 0.65 \\
\hline$V_{\mathrm{T}}$ expiration $\mathrm{mL}$ & $440 \pm 14$ & $453 \pm 15$ & 0.56 & $420 \pm 26$ & $404 \pm 32$ & 0.72 \\
\hline Peak pressure $\mathrm{cmH}_{2} \mathrm{O}$ & $15 \pm 1$ & $19 \pm 1$ & 0.01 & $15 \pm 1$ & $17 \pm 1$ & 0.32 \\
\hline Air leakage $\mathrm{mL}$ & $13 \pm 2$ & $21 \pm 1$ & 0.31 & $16 \pm 4$ & $14 \pm 7$ & 0.79 \\
\hline
\end{tabular}

Data are presented as mean \pm SEM, unless otherwise stated. BP: blood pressure; MAP: mean arterial pressure; $\mathrm{EtCO}_{2}$ : end tidal carbon dioxide; $V_{\mathrm{T}}$ : tidal volume. 
facilitate the exchange of oxygen tension and carbon dioxide tension. It has been suggested previously that PEx are derived from the opening and closing of the distal airways and alveoli [3]. Our results indicate that mechanically ventilated patients might have lesser opening and closure of the distal airways compared with NB patients. NB patients most likely promote an opening and closure of the distal airways to a higher extent then mechanically ventilated patients and the PExA device is able to detect these differences.

\section{Biochemical collection achievable in mechanically ventilated patients during surgery}

In addition to differences in PFR, we also sought to further understand the composition of the PEx as this most likely correlates with their origin. We looked at expression levels of DPPC and POPC which are major components of SP-A along with albumin. They are all believed to originate from the RTLF with albumin thought to be more universally expressed along the airway lining fluid while SP-A is predominantly being produced by alveolar epithelial cells and secreted into the alveolar space. DPPC and POPC were detected in all samples and showed significantly lower levels for mechanically ventilated patients compared to NB. The opening and closure of the distal airways are likely to be more prominent in the NB group compared with mechanically ventilated patients due to differences in their breathing. We could also see lower concentrations of DPPC and POPC in MV-C and MV-NSCLC, compared with NB. This indicates that that PEx from NB more prominently represents the distal airways as compared to mechanically ventilated patients.

We were able to detect both albumin and SP-A in a subset of patients undergoing surgery and were able to detect albumin and SP-A in all NB patients. Interestingly, we found that MV-NSCLC had lower levels of SP-A levels (both with regard to wt. \% of total PEx and albumin/SP-A ratio) compared with NB, but that there were no detectable differences for albumin. These findings are also most likely due to the fact that they stem from differences in particle origin and supports the fact that different breathing patterns generate particles with a different composition which most likely is due to differences in the PEx origin from different parts of the airways. NB patients' PEx most likely originate predominantly from the distal airways compared with mechanically ventilated patients. Importantly, our NB controls were patients who also had NSCLC, confirming that differences observed between NSCLC patients and NB patients were likely because of sampling method and not due to differences caused by disease.

However, several additional factors might alter and influence the flow and the composition of particles. We have shown previously in animal models that blood flow and different ventilation modes, i.e. volume-controlled and pressure-controlled ventilation, alter PFR from the airways $[14,15]$. We believe that in this study, the magnitude of the difference we observed was primarily due to the degree of opening and closing of the distal airways and that this is the major factor influencing the particle flow and its composition.

\section{Opening up previous closed airways will generate higher PFR}

The measurements stopped at the end of surgery when the patients were still on OLV but in nine patients, the surgery allowed for additional measurements when transiting from OLV to DLV. Our findings demonstrate that by going from ventilating by OLV to DLV, i.e. opening up a previously closed lung, a significant increase in PFR was detected but there was no change in average particle mass per particle. We believe that these results are in line with the results of this and other studies that support the notion that opening and closing of the distal airways results in increased PFR in exhaled air and that the effect can be detected with this technique $[14,15]$. The fact that there is no significant difference in average particle mass between OLV and DLV is supportive of the concept that mechanical ventilation induces particles with similar mass profiles but that PFR is increased due to the fact that there are more accessible airways in the DLV scenario. In further support of the concept that particle flow is related to the opening and close of distal airways, we found that there were no significant differences between MV-NSCLC and MV-C (i.e. mechanical ventilation) with regard to both total PFR and particle mass, but that there were significant differences in both of these measurements when compared with NB patients. Therefore, our data suggest that an opening and closing of the airways induces PEx.

\section{The PEXA instrument is safe to use in intubated mechanically ventilated patients during surgery}

This study was performed in intubated surgical patients during mechanical ventilation and was performed as a feasibility study. No adverse events were observed. In the MV-NSCLC patient group, we observed a significant difference in peak pressure at the end of the measurement period as compared to the start, as seen in table 2. The peak pressure at the end of the measurement is well below any known risk level for a patient. In conclusion, we did not detect any harmful effects on all the intubated patients using PExA, and we believe this technique can safely be used for intubated patients on mechanical ventilation during surgery. The findings from this study and from previous animal studies are encouraging and indicate that the PExA method might be a novel tool to give new insight to lung physiology during mechanical ventilation $[14,15]$. 
Potential and interesting future direction for this noninvasive, direct and on-line technique during mechanical ventilation could be in optimising ventilation during surgery and do so in real-time. It is a tempting conclusion that the on-line information from the PExA instrument could contribute to strive towards a gentler mechanical ventilation and thereby reduce the risk for lung injury both during surgery but also in intensive care. A sudden increase or high PFR may strongly imply an increased opening and closing of the distal airways which is known to be harmful to the lung tissue [18-21]. On-line monitoring of PFR might therefore be useful in modifying mechanical ventilation settings to induce less harm to the lung tissue and due so on an individual level adapted to each patient.

\section{Limitations}

There is a hypothetical possibility of particle deposition on the inside of the endotracheal tube or other parts of the respiratory circuit, which has not been studied; however, all patients were exposed to the same equipment with the same lengths of respiratory collection circuit. In the present study, we only study particles within the detectable range (diameter range of $0.41-4.55 \mu \mathrm{m}$ ) although the majority of the particles originated from the RTLF are most likely within this range.

\section{Conclusions}

We have established the safety and feasibility of the PExA device during surgery. Our study showed higher PFR from patients breathing normally as compared to patients on mechanical ventilation, independently from surgical indication. Higher PFR is thought to be generated when opening and closing of the airway is more frequent, something known to be harmful to the lung. We believe by using the PExA device online, individual and more optimal alteration of mechanical ventilation settings could be used and thereby lower the risk for lung damage. Furthermore, particles in exhaled air can be collected during mechanical ventilation and analysed for phospholipids and proteins. We believe this technology will be useful to strive towards more gentle ventilation by optimising ventilator settings during surgery and the technology also has the potential to detect biomarkers in exhaled air.

Acknowledgements: The authors thank Markus Ståhlman (Wallenberg Laboratory) and Hatice Koca Akdeva, at Sahlgrenska Academy, University of Gothenburg, Gothenburg, Sweden, for the assistance with the biochemical analysis.

Author contributions: S. Lindstedt, E. Broberg, J. Andreasson and S. Hyllén participated in the design of the study. S. Lindstedt and E. Broberg wrote the application for ethical approval. J. Andreasson operated on the patients. E. Broberg and S. Hyllén performed the PExA measurements in the intubated patients. M. Fakhro and J. Andreasson carried out the PExA measurements on the normal breathing patients. A-C. Olin carried out analyses of surfactant protein (SP)-A and albumin. S. Lindstedt, E. Broberg, M. Fakhro and D. Wagner analysed the study results regarding particle flow, particle count and mass, and SP-A and albumin. S. Lindstedt and E. Broberg drafted the manuscript. All authors read and approved the final manuscript.

Conflict of interest: E. Broberg has nothing to disclose. J. Andreasson has nothing to disclose. M. Fakhro has nothing to disclose. A-C. Olin reports that she is a board member and shareholder of PExA AB, and has a patent (wo2009045163) licenced to PExA AB. D. Wagner reports a Wallenberg Molecular Medicine Fellowship during the conduct of the study; and speaker's honoraria from Boehringer Ingelheim, an ERC Starting Grant and a Swedish Research Council Starting Grant, outside the submitted work. S. Hyllén has nothing to disclose. S. Lindstedt reports a Wallenberg Molecular Medicine Fellowship, and grants from the Sjöberg Foundation and the ALF foundation, during the conduct of the study.

Support statement: This work was funded by the Sjöberg Foundation (S. Lindstedt), a Wallenberg Molecular Medicine Fellowship (S. Lindstedt and D. Wagner) from the Knut and Alice Wallenberg Foundation, and the ALF foundation (S. Lindstedt). Funding information for this article has been deposited with the Crossref Funder Registry.

\section{References}

1 Bredberg A, Gobom J, Almstrand AC, et al. Exhaled endogenous particles contain lung proteins. Clin Chem 2012; 58: 431-440.

2 Larsson P, Bake B, Wallin A, et al. The effect of exhalation flow on endogenous particle emission and phospholipid composition. Respir Physiol Neurobiol 2017; 243: 39-46.

3 Bake B, Larsson P, Ljungkvist G, et al. Exhaled particles and small airways. Respir Res 2019; 20: 8.

4 Scheideler L, Manke HG, Schwulera U, et al. Detection of nonvolatile macromolecules in breath. A possible diagnostic tool? Am Rev Respir Dis 1993; 148: 778-784.

5 Griese M. Pulmonary surfactant in health and human lung diseases: state of the art. Eur Respir J 1999; 13: $1455-1476$.

6 Koetzler R, Saifeddine M, Yu Z, et al. Surfactant as an airway smooth muscle relaxant. Am J Respir Cell Mol Biol 2006; 34: 609-615.

7 Chiba H, Piboonpocanun S, Mitsuzawa H, et al. Pulmonary surfactant proteins and lipids as modulators of inflammation and innate immunity. Respirology 2006; 11: Suppl., S2-S6.

8 Horvath I, Barnes PJ, Loukides S, et al. A European Respiratory Society technical standard: exhaled biomarkers in lung disease. Eur Respir J 2017; 49: 1600965.

9 Almstrand AC, Bake B, Ljungstrom E, et al. Effect of airway opening on production of exhaled particles. J Appl Physiol 2010; 108: 584-588. 
10 Almstrand AC, Ljungstrom E, Lausmaa J, et al. Airway monitoring by collection and mass spectrometric analysis of exhaled particles. Anal Chem 2009; 81: 662-668.

11 Beck O, Olin AC, Mirgorodskaya E. Potential of mass spectrometry in developing clinical laboratory biomarkers of nonvolatiles in exhaled breath. Clin Chem 2016; 62: 84-91.

12 Ericson PA, Mirgorodskaya E, Hammar OS, et al. Low levels of exhaled surfactant protein A associated with BOS after lung transplantation. Transplant Direct 2016; 2: e103.

13 Larsson P, Larstad M, Bake B, et al. Exhaled particles as markers of small airway inflammation in subjects with asthma. Clin Physiol Funct Imaging 2017; 37: 489-497.

14 Broberg E, Wlosinska M, Algotsson L, et al. A new way of monitoring mechanical ventilation by measurement of particle flow from the airways using Pexa method in vivo and during ex vivo lung perfusion in DCD lung transplantation. Intensive Care Med Exp 2018; 6: 18.

15 Broberg E, Pierre L, Fakhro M, et al. Different particle flow patterns from the airways after recruitment manoeuvres using volume-controlled or pressure-controlled ventilation. Intensive Care Med Exp 2019; 7: 16.

16 Larstad M, Almstrand AC, Larsson P, et al. Surfactant protein A in exhaled endogenous particles is decreased in chronic obstructive pulmonary disease (COPD) patients: a pilot study. PLoS One 2015; 10: e0144463.

17 Larsson P, Mirgorodskaya E, Samuelsson L, et al. Surfactant protein A and albumin in particles in exhaled air. Respir Med 2012; 106: 197-204.

18 Della Rocca G, Coccia C: Acute lung injury in thoracic surgery. Curr Opin Anaesthesiol 2013; 26: 40-46.

19 Licker M, Diaper J, Villiger Y, et al. Impact of intraoperative lung-protective interventions in patients undergoing lung cancer surgery. Crit Care 2009; 13: R41.

20 Serpa Neto A, Hemmes SN, Barbas CS, et al. Protective versus conventional ventilation for surgery: a systematic review and individual patient data meta-analysis. Anesthesiology 2015; 123: 66-78.

21 Silva PL, Negrini D, Rocco PR. Mechanisms of ventilator-induced lung injury in healthy lungs. Best Pract Res Clin Anaesthesiol 2015; 29: 301-313. 